$\xi=-1$

\title{
Efficient spectrum sensing technique based on energy detector, compressive sensing, and de-noising techniques
}

\author{
Amr H. Hussien, Hossam M. Kasem, Mohamed A. Ezzat * \\ Electronics and Electrical Communications Department, Faculty of Engineering, Tanta University, Tanta Egypt \\ *Corresponding author E-mail: mohamed.radwan@f-eng.tanta.edu.eg
}

\begin{abstract}
Highdata rate cognitive radio (CR) systems require high speed Analog-to-Digital Converters (ADC). This requirement imposes many restrictions on the realization of the CR systems. The necessity of high sampling rate can be significantly alleviated by utilizing analog to information converter (AIC). AIC is inspired by the recent theory of Compressive Sensing (CS), which states that a discrete signal has a sparse representation in some dictionary, which can be recovered from a small number of linear projections of that signal. This paper proposes an efficient spectrum sensing technique based on energy detection, compression sensing, and de-noising techniques. De-noising filters are utilized to enhance the traditional Energy Detector performance through Signal-to-Noise (SNR) boosting. On the other hand, the ordinary sampling provides an ideal performance at a given conditions. A near optimal performance can be achieved by applying compression sensing. Compression sensing allows signal to be sampled at sampling rates much lower than the Nyquist rate. The system performance and ADC speed can be easily controlled by adjusting the compression ratio. In addition, a proposed energy detector technique is introduced by using an optimum compression ratio. The optimum compression ratio is determined using a Genetic Algorithm (GA) optimization tool. Simulation results revealed that the proposed techniques enhanced system performance.
\end{abstract}

Keywords: Cognitive Radio (CR); Compressive Sensing; De-Noising Filters; Genetic Algorithm (GA); Spectrum Sensing (SS).

\section{Introduction}

There is a large demand on high data rate wireless services over spectrum-based communications. Because of the inefficient utilization of the allowed spectrum, unlicensed bands should be utilized without making interference with the licensed bands. Cognitive radio systems have become the most reliable system to utilize these unlicensed bands depending on spectrum sensing. Spectrum sensing (SS) offers the Secondary Users (SU) an access to these unlicensed bands without making an interference with the Primary Users (PU). Energy detection is the most widely used technique for spectrum sensing because of its low computational and implementation complexities as mentioned in [1]. On the other hand, energy detection suffers of the poor performance under low SNR scenarios as presented in [2]. Hybrid Slantlet transforms (HST) has been used in [3] and [4], as a de-noising technique to solve the noise impact alleviation. Moreover, HST is a set of digital filters, which separate the noise from the non-stationary PU signals.

$\mathrm{CS}$ is an acquisition method that can enable sampling sparseor compressible signals in some basis, at a rate much lower than Nyquist-rate [5-6]. CS found its way to digital multimedia, including video, medical imaging and high-quality speech as well as wide-band communication applications [7].

Sensing of a wideband spectrum is a very challenging problem due to its high sampling rate requirements. This led to a complex and an expensive hardware problem. CS is introduced to reduce the number of samples required to acquire the spectrum, by exploiting the unique sparsity in the wide-band spectrum.

In this paper, a new high performance spectrum sensing technique is introduced. It is based on ED, CS, and De-noising techniques. CS is utilized to reduce the number of samples required to acquire the spectrum. On the other hand, De-noising filters, such as Recursive Lease Square (RLS) and Wavelet filters, are used to increase SNR of the received signal. Simulation results show that utilization of de-noising filters is significantly enhanced the Receiver Operating Characteristics (ROC) curves of the CR system. In addition, the utilization of CS provides a close performance compared to traditional Nyquist sampling system. This paper is organized as follows. In section 2, spectrum sensing and traditional energy detector are briefly described. The CS and its recovery algorithms are briefly introduced in section 3 . The de-noising filters are described in section 4 . The proposed spectrum sensing technique is introduced in section 5. Finally, simulation results are discussed in section 6 .

\section{Spectrum sensing and energy detector}

This part introduces a brief background on ED. First a primary signal is considered as $\mathrm{s}(\mathrm{n})$. The primary signal has a carrier frequency $\mathrm{f}_{\mathrm{c}}$ with a bandwidth of $\mathrm{W} \mathrm{Hz}$, which can be detected by a secondary users' receiver. Then, the signal received at the receiver expressed as:

$y(n)=s(n)+u(n)$

Wheres(n)denotes the detected signal, $\mathrm{u}(\mathrm{n})$ is the Additive White Gaussian Noise (AWGN). AWGN has a mean of $\mu_{u}=0$ and variance $\sigma_{u}^{2}$, and $n$ is the sample index. The primary user is detected between a two hypotheses. The two hypotheses are the absence of the received primary signal and the presences of the primary signal which denoted as $\mathrm{H}_{\mathrm{o}}$ and $\mathrm{H}_{1}$ respectively. The two mathematical hypotheses are expressed in the following equations: 
$H_{o}: y(n)=u(n)$

$H_{1}: y(n)=s(n)+u(n)$

Energy in primary signal is measured through ED. The differentiation between the above two hypotheses is calculated through a decision metric equation expressed as:

$\mathrm{M}(\mathrm{y})=\frac{1}{\mathrm{~N}} \sum_{\mathrm{n}=1}^{\mathrm{N}}|\mathrm{y}(\mathrm{n})|^{2}$

By assuming $\tau$ to be sensing time and $\mathrm{N}$ is a total number of samples, the relation between them is expressed by the following equation:

$\mathrm{N}=\tau \mathrm{f}_{\mathrm{s}}$

The decision metric $\mathrm{M}(\mathrm{y})$ is a random variable which has a Probability Density Function (PDF). For a large number of samples N according to [8], usually $\mathrm{N} \geq 250$, a Gaussian approximation is used to the PDF of test statistics M(y) under either hypothesis: Ho noise alone, or $\mathrm{H}_{1}$ signal with noise. This is done by using Central Limit Theorem (CLT) [8].

The probability of an algorithm correctly detects the presence of a primary signal is called probability of detection $\left(P_{d}\right)$, and the probability of an algorithm falsely declares the presence of a primary signal is called probability of false alarm $\left(\mathrm{P}_{\mathrm{f}}\right)$ under hypothesis $\mathrm{H}_{1}$ and $\mathrm{H}_{\mathrm{o}}$ respectively [8]. The probability of false alarm is given by:

$\mathrm{P}_{\mathrm{f}}(\epsilon, \tau)=\mathrm{Q}\left(\left(\frac{\epsilon}{\sigma_{\mathrm{u}}^{2}}-1\right) \sqrt{\tau \mathrm{f}_{\mathrm{s}}}\right)$

Probability of detection can be approximated by:

$\mathrm{P}_{\mathrm{d}}(\epsilon, \tau)=\mathrm{Q}\left(\left(\frac{\epsilon}{\sigma_{\mathrm{u}}^{2}}-\gamma-1\right) \sqrt{\frac{\tau \mathrm{f}_{\mathrm{s}}}{2 \gamma+1}}\right)$

Where $Q(\cdot)$ is the complementary distribution function of the standard Gaussian, according to [6] i.e.

$Q(x)=\frac{1}{\sqrt{2 \pi}} \int_{x}^{\infty} \exp \left(-\frac{t^{2}}{2}\right) d t$

$\gamma=\frac{\sigma_{\mathrm{s}}^{2}}{\sigma_{\mathrm{u}}^{2}}$

Where $\sigma_{\mathrm{s}}^{2}$ is signal variance and $\gamma$ is SNR. The probability of missed detection can be written as:

$\mathrm{P}_{\mathrm{md}}=1-\mathrm{P}_{\mathrm{d}}$

From equation (6), detection threshold $\epsilon$ is related to the probability of false alarm as follows:

$\epsilon=\left(\frac{\mathrm{Q}^{-1}\left(\mathrm{P}_{\mathrm{f}}\right)}{\sqrt{\tau \mathrm{f}_{\mathrm{s}}}}+1\right) \sigma_{\mathrm{u}}^{2}$

As shown in Fig. 1, the energy detector consists of three main parts. The first part is noise pre-filter which is used to limit noise bandwidth. The second part is squaring device which is used to calculate the energy of the filtered signal. Finally, the third part is an integrator which determines the energy of the filtered received signal over the sensing time interval $\tau$. The integrator output is considered to be the test statistic of the two hypothesis $\mathrm{H}_{\mathrm{o}}$ and $\mathrm{H}_{1}$.

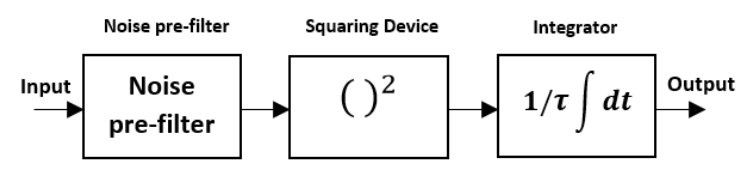

Fig. 1: Energy Detector Simplified Model.

\section{Compressive sensing}

The theory of CS was developed by Donho and Cande's [9], [10]. In a traditional sampling theorem, the signal is sampled using Nyquist rate, whereas with the help of CS the signal is sampled below the Nyquist rate. This is possible because the signal is transformed into a domain in which the signal has a sparse representation. Then the signal is reconstructed from the samples by exploiting the sparsity property [11], [12].

\subsection{Signal representation and sparsity}

Suppose there is a basis $\Psi$ (i.e. inverse Wavelet Transform (WT), inverse Discrete Cosine Transform (DCT) and inverse Discrete Fourier Transform (DFT)) in which real value signal $\mathrm{X}$ is sparse. The real-valued signal can be represented as [9], [10]:

$X=\Psi f$

Where $\Psi$ is $\mathrm{N} \times \mathrm{N}$ matrix whose columns are orthonormal basis functions, and $\mathrm{f}$ is sparse coefficients vector. If there are only $\mathrm{k}$ non-zero coefficients in the vector $\mathrm{f}$, we can say that $\mathrm{X}$ is $\mathrm{k}$ sparse. According to the CS theory [9], [10], state that X can be accurately recovered from $\mathrm{M}$ random measurements over the measurement matrix according to (13) [9], [10].

$\mathrm{y}_{\mathrm{cs}}=\Phi \mathrm{X}$

Where $\mathrm{y}_{\text {cs }}$ is a $\mathrm{M}$ dimensional measurement vector with $\mathrm{M} \ll$ $\mathrm{N}$ and $\Phi$ is $\mathrm{M} \times \mathrm{N}$ random measurement matrix which represents the measurement process. Typically $M \geq$ const $\times \operatorname{klog}\left(\frac{N}{k}\right)$ [9], [10]. Substituting (12) into (13):

$\mathrm{y}_{\mathrm{cs}}=\Psi \Phi \mathrm{f}=\mathrm{Af}$

Where $A=\Psi \Phi$ the sensing matrix is typically full rank.

\subsection{Recovery algorithms for CS}

By examining the compressed sensing equation:

$\mathrm{y}_{\mathrm{cs}}=\mathrm{Af}$

The direct solution involves solving $\mathrm{M}$ equation in $\mathrm{N}$ unknowns.This led to determination solution set. In order to solve this ambiguity, the sparsity should be manipulated in the recovery algorithms of the sparse signal $\mathrm{f}$.

Let $\mathrm{f}$ now be a sparse vector. It is quite intuitive to recover $\mathrm{f}$ from knowledge of $\mathrm{y}_{\mathrm{cs}}$ by solving:

$\hat{\mathrm{f}}=\operatorname{argmin}\|\mathrm{f}\|_{0}$

s. $\mathrm{y}_{\mathrm{cs}}=\mathrm{Af}$

Where the zeroth order norm $\|.\|_{0}$ measures the number of nonzero values in a vector and it is the faithful representation of the sparsity order. However, this measure is nonlinear and the recovery algorithm in this case in NP-hard [10], [13]. The main idea of Chen, Donoho, and Saunders [9] was to substitute the $\mathrm{l}_{\mathrm{o}}$ norm by the closest convex norm, which is the $l_{1}$ norm. This leads to the following minimization problem, which called Basis Pursuit (BP) [9], [10]: 
$\hat{\mathrm{f}}=\operatorname{argmin}\|\mathrm{f}\|_{1}$

s.t $y_{c s}=A f$

There are many types of recovery algorithms, which can be suggested. They can, generally, be classified into greedy algorithms and convex optimization based algorithms. Greedy algorithms iteratively approximate the coefficients and the support of the original signal. They have the advantage of being very fast and easy implementation. The simplest effective greedy algorithm is used. This algorithm called Orthogonal Matching Pursuit (OMP) [14], [15].

OMP is one of the earliest methods for sparse approximation. Basic references for this method in the signal processing literature are introduced in [14] and, [15]. However, the idea has appeared for the first time in variable selection in regression in [15].

Another fundamental family of algorithms for sparse recovery replaces the combinatorial $l_{o}$ function with the $l_{1}$-norm, yielding convex optimization problems. In a concrete sense [16], the $l_{1}$ norm is the closest convex function to the $l_{o}$ function, so this relaxation is quite natural.

The proposed algorithm formulates (16) in convex optimization form [17]. This led to enabling the use of many available well developed optimization packages. One possibility to solve (16) is to use CVX package [18], [19].

\section{De-noising filters}

In this section, two powerful de-noising filters are briefly introduced to reduce the effect of the noise on the received signal.

\subsection{Recursive least square filter}

Recursive Least Square (RLS) filter is an adaptive filter. Its frequency response is adjustable to enhance its performance according to some criterion. This allows the RLS filter to adapt and change. RLS filter consist of two parts: the first part is a digital filter with adjustable coefficients. The second part is an adaptive algorithm, which can modify filter coefficients [20], [21].

An adaptive filter consists of two distinct parts: a digital filter with adjustable coefficients, and an adaptive algorithm which is used to adjust or modify the coefficients of the filter [8] and [20]. RLS can be briefly modeled as in Fig. 2 [8].

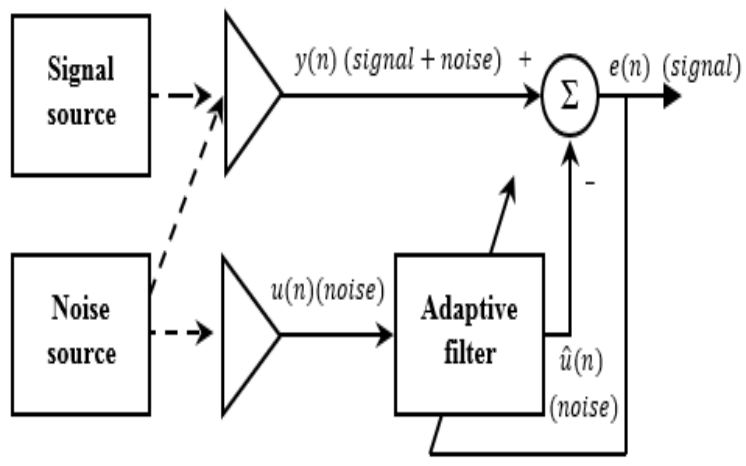

Fig. 2: RLS Filter Model [8].

From Fig.2, two input signals $y(n)$ and $u(n)$ are applied simultaneously to an adaptive filter where, $y(n)$ is a contaminated signal containing both uncorrelated desired signal $s(n)$ and noise $u(n)$. The main objective of the adaptive algorithm is to produce an optimum estimation of noise $\hat{u}(n)$ in the contaminated signal. This can be achieved by using a suitable adaptive algorithm to minimize noise [20]. A simple equation of the RLS filter can be expressed as:

$e(n)=y(n)-\hat{u}(n)=s(n)+u(n)-\hat{u}$
Where $e(n)$ is the error signal, which has two purposes; the first one is to estimate the desired signal and the second is to adjust filter coefficients.

\subsection{Wavelet de-noising filter}

Wavelet is defined as wave-like oscillation with amplitude starts out at zero, increase, and then decreases back to zero. In statistics, recovering an underlying function from a noisy signal always modeled using regression model. This method is called Wavelet de-noising by Thresholding. Suppose that there is a number of $n$ noisy samples of a function $s$.

$y_{i}=s\left(t_{i}\right)+\sigma u(n), i=1 \ldots n$

Where $y$ is the noisy data, $y=\left(y_{1}, \ldots, y_{n}\right)$, and $u(n)$ is AWGN, $N\left(0, \sigma_{u}^{2}\right)$ and the noise level $\sigma$ is unknown. The main goal is to recover function $s$ from the noisy data $y_{i}$ that satisfies the following equation:

$$
\hat{s}=\min _{\hat{s}}\|\hat{s}-s\|_{2}
$$

Where $\hat{s}=\hat{s}(y)$. the thresholding of the wavelet coefficients is usually applied only to the detail coefficients of $y$ rather than the approximation coefficients. Because the approximation coefficients always contain the most important components of the signal, and are less affected by noise. Thresholding will set significant coefficients, whose values will be below a certain threshold level $\lambda$, to zero [23].

\section{Proposed spectrum sensing technique}

In this section, an efficient spectrum sensing technique based on de-noising filters and CS is introduced. Fig.3 shows the block diagram of the proposed technique. CS allows signal sampling at sampling rates much lower than the nyquist rate. In this case, lower speed ADCs can be utilized. Furthermore, the system performance and ADC speed can be easily controlled by adjusting the compression ratio.

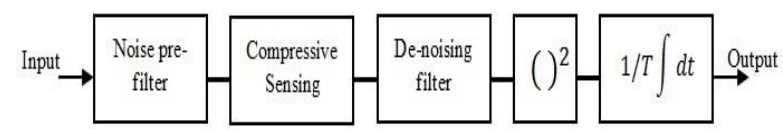

Fig. 3: Block Diagram of the Proposed Spectrum Sensing Technique.

First the input signal is passed through a noise pre-filter. Then the signal is applied to CS technique with different compression ratios. CS will reduce the number of samples needs to reconstruct the original signal at the receiver. De-noising filter will minimize the added noise of the received signal which leads achieving SNR gain and variance reduction. Consequently, the improvement will enhance the detection threshold estimation and improve the ROC curves of the energy detector. Enhancing ROC curves is because of the increment in probability of detection $P_{d}$ and the decrement in probability of false alarm $P_{f}$ and probability of missed detection $P_{m d}$.

\section{Throughput calculatioins}

In order to compare between the performance of the proposed technique and the traditional energy detector, the achievable sensing throughput is calculated.In order to evaluate cognitive radio networks under noise uncertainty is through sensing throughput calculations as mentioned in [24].According to [22], a Cognitive radio network has a frame structure with number of frames equal 
to Nas shown in the Fig.4. The achievable sensing throughput is function of sensing time $\tau$.

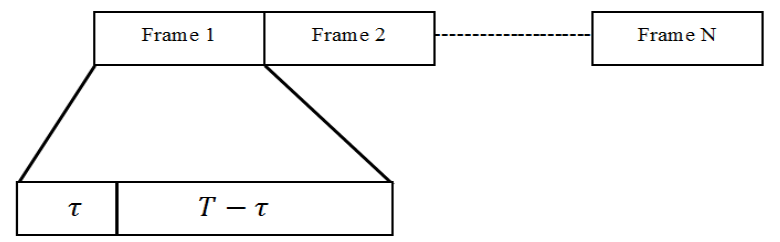

Fig. 4: Frame Structure [6].

Two time slots, sensing time slot $\tau$ and data transmission slot (T$\tau$ ) are shown on the frame structure [25]. Primary user could be absent or presented. In the absence of primary user, sensing throughput is calculated as below:

$C_{o}=\log _{2}\left(1+S N R_{S}\right)=\log _{2}\left(1+\frac{P_{S}}{N_{o}}\right)$

Where $C_{o}$ is the throughput of secondary network in the absence of the primary user, $S N R_{S}=P_{S} / N_{o}$ is signal to noise ratio of the secondary network where and $P_{S}$ is the received power of the secondary network. On the other hand, sensing throughput is calculated in the presence of the primary user from the equation below:

$C_{1}=\log _{2}\left(1+\frac{S N R_{s}}{1+S N R_{p}}\right)=\log _{2}\left(1+\frac{P_{S}}{P_{p}+N_{o}}\right)$

Where $C_{1}$ is the secondary network throughput when the primary user is present, and $S N R_{p}=P_{p} / N_{o}$ is signal to noise ratio of the secondary network, where $P_{p}$ is the interference power of the primary user.

Obviously from equations (21) and (22), it is found that $C_{o}>C_{1}$. For a given frequency band, the probability for which the primary user is present is defined as $P\left(H_{1}\right)$ and the probability for which the primary user is absence is defined as $P\left(H_{o}\right)$ where:

$P\left(H_{o}\right)+P\left(H_{1}\right)=1$

Now achievable sensing throughput can be calculated from the equation below:

$\tilde{R}(\tau)=C_{o} P\left(H_{o}\right)\left(1-\frac{\tau}{T}\right)\left(1-Q\left(\alpha+\sqrt{\tau f_{S} \gamma}\right)\right)$

Where $\alpha=\sqrt{2 \gamma+1} Q^{-1}\left(P_{d}\right)$. In IEEE 802.22 WRAN [22], probability of detection is chosen where $P_{d}=0.9$ for $S N R=-20 \mathrm{~dB}$, and the activity probability $P\left(H_{1}\right)=0.2$ for the primary user.

\section{Simulation results}

In this section, a performance comparison between the proposed spectrum sensing technique and the traditional energy detector is presented. Simulations are performed using MATLAB R2014a. A frame of 8 bits is sent using BPSK modulation with bandwidth of $2 \mathrm{MHz}$. AWGN is added to a modulated signal for SNR equals $-20 d B$. Three test cases are discussed. The first case is intended to apply the de-noising techniques on the ordinary sampled received signal in order to study the performance enhancement of the energy detector. In the second case, the de-noising techniques are applied on the compressive sensing reconstructed received signal at different compression ratios. The effect on the performance of the energy detector is studied. In the third case, a simulation result of the proposed energy detector technique using an optimization compression ratio is introduced. The optimum compression ratio is determined using the Genetic Algorithm (GA) optimization tool.
Case 1: Applying de-noising techniques on ordinary sampled signal

In this part, both RLS and wavelet De-noising filters are applied on the received signal which is ordinary sampled at Nyquist rate to study the performance enhancement of the energy detector. The simulation results revealed that the de-noising techniques are, greatly, enhanced the performance of the energy detector. On the other hand, the energy detector based on RLS de-noising filter provided a much higher performance more than the energy detector based on wavelet de-noising filter and the traditional energy detector as shown in Fig.5 and Fig.6.

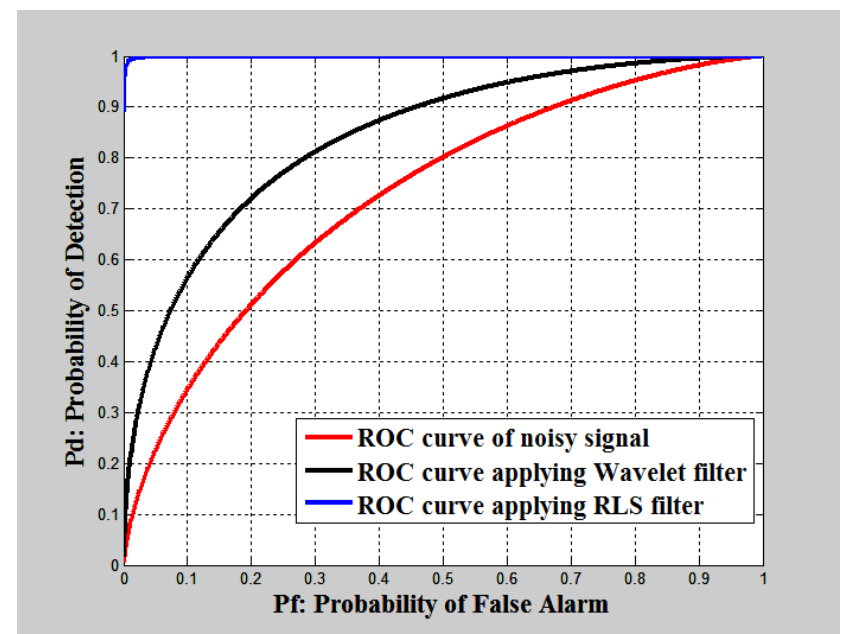

Fig. 5: Comparison Between ROC Curves for the Proposed Energy Detectors Based on De-Noising Filters and the Traditional Energy Detector Using Ordinary Sampling.

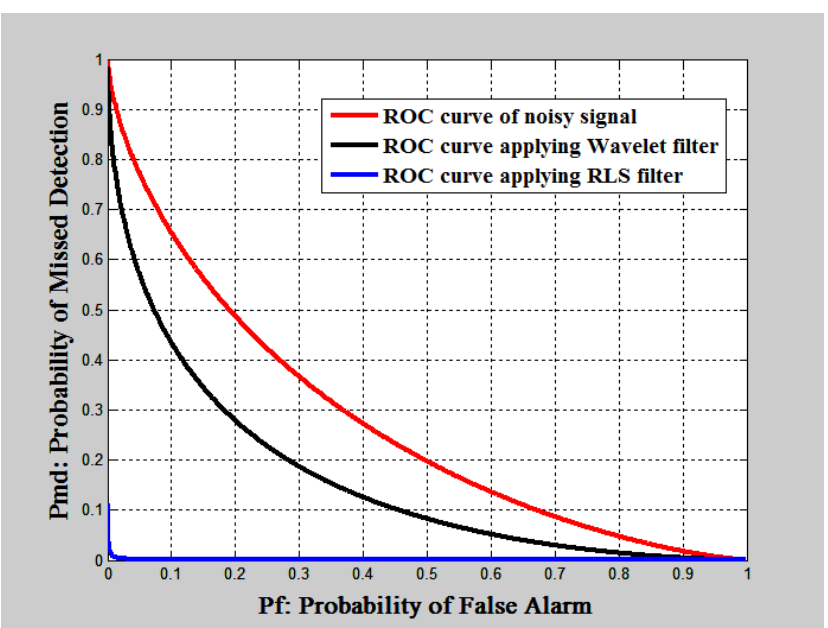

Fig. 6: Comparison Between ROC Curves for the Proposed Energy Detectors Based on De-Noising Filters and the Traditional Energy Detector Using Ordinary Sampling.

In addition, the performance enhancement effect of the energy detector has greatly reflected on the throughput of the system. For a certain probability of detection $P_{d}=0.9, T=100 \mathrm{~ms}, \tau=$ $0.4 \mu s$, and $S N R_{p}=100$, the calculated throughputsfor the traditional energy detector and the proposed energy detectors are listed in table 1. Throughput calculations show that after applying the wavelet and RLS de-noising filters, the throughputs are increased to 0.038 and $0.15 \mathrm{bits} / \mathrm{sec} / \mathrm{Hz}$ which are 3.166 and 12.5 times greater than the throughput of the traditional detector respectively. It is clear that the RLS de-noising filter provides the highest throughput as shown Fig.7. 
Table 1: Throughput Calculations for the Proposed Energy Detectors Using Ordinary Sampling.

\begin{tabular}{|c|c|c|c|c|c|c|c|}
\hline & $P_{d}$ & $P_{f}$ & $\tau \mu \mathrm{s}$ & $\begin{array}{l}\mathrm{T} \\
\mathrm{ms}\end{array}$ & $\mathrm{SNR}_{\mathrm{s}}$ & $\mathrm{SNR}_{\mathrm{p}}$ & $\begin{array}{l}\text { Throughput } \\
\text { bits/sec/Hz }\end{array}$ \\
\hline $\begin{array}{l}\text { Traditional } \\
\text { Energy } \\
\text { Detector }\end{array}$ & 0.9 & 0.66 & 0.4 & 100 & 0.03 & 100 & 0.012 \\
\hline $\begin{array}{l}\text { Proposed } \\
\text { detector } \\
\text { based on } \\
\text { wavelet de- } \\
\text { noising } \\
\text { filter }\end{array}$ & 0.9 & 0.40 & 0.4 & 100 & 0.06 & 100 & 0.038 \\
\hline $\begin{array}{l}\text { Proposed } \\
\text { detector } \\
\text { based on } \\
\text { RLS de- } \\
\text { noising } \\
\text { filter }\end{array}$ & 0.9 & 0.012 & 0.4 & 100 & 0.13 & 100 & 0.15 \\
\hline
\end{tabular}

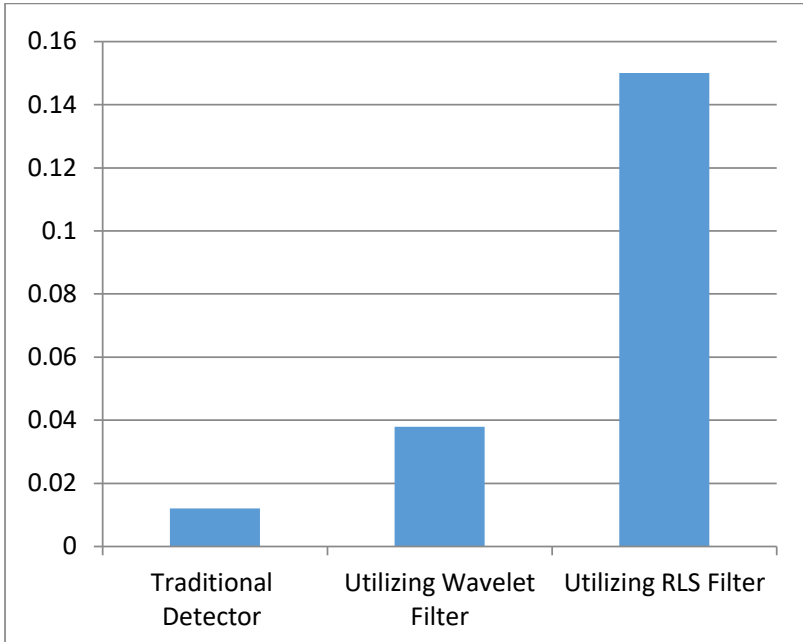

Fig. 7: Comparison between Throughputs for the Energy Detectors Based On De-Noising Filters and the Traditional Energy Detector Using Ordinary Sampling.

Case 2: Applying de-noising techniques on compressed signal using compressive sensing

The De-noising techniques are applied on the received signal which is constructed by compressive sensing at the compression ratios 20\%, 40\%, 60\%, and 80\%. Fig. 8 through Fig. 15 show the ROC curves of the proposed detector using compressive sensing compared to the energy detector using ordinary sampling. The simulation results indicate that as the compression ratio increase the ROC curves using CS approach the ROC curves using the ordinary sampling. For a probability of detection $\mathrm{P}_{\mathrm{d}}=0.9, \mathrm{~T}=$ $100 \mathrm{~ms}, \tau=0.4 \mu \mathrm{s}$, and $\mathrm{SNR}_{\mathrm{p}}=100$, the corresponding throughputs are calculated as shown in Fig.16. It is clear that the throughputs are lower than the optimal throughput achieved using ordinary sampling. But, it is worth noting that for compression ratios greater than $60 \%$, the curves using CS become very close to the ROC curves using the ordinary sampling.

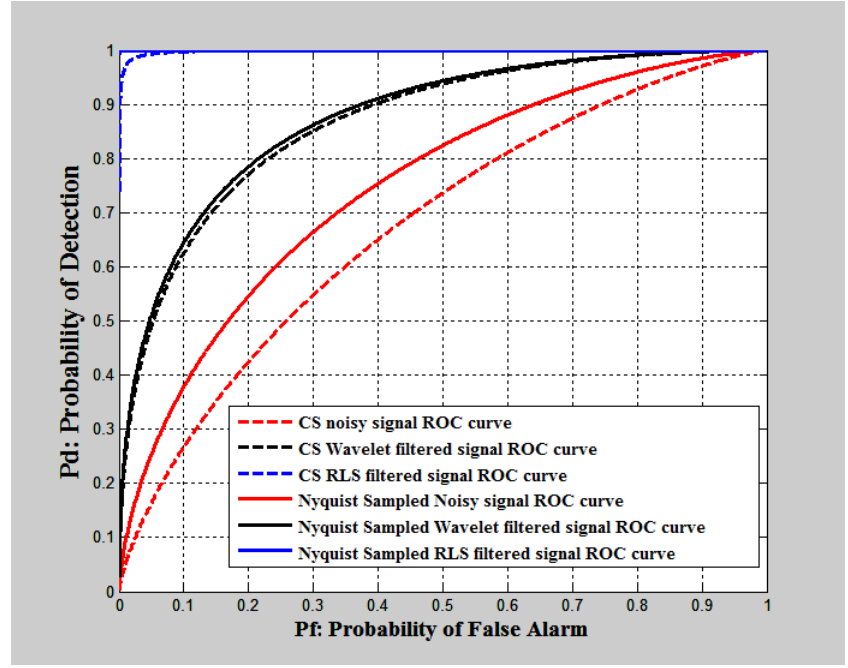

Fig. 8: Comparison between ROC Curves at 20\% Compression Ratio and the Traditional Energy Detector Using Ordinary Sampling.

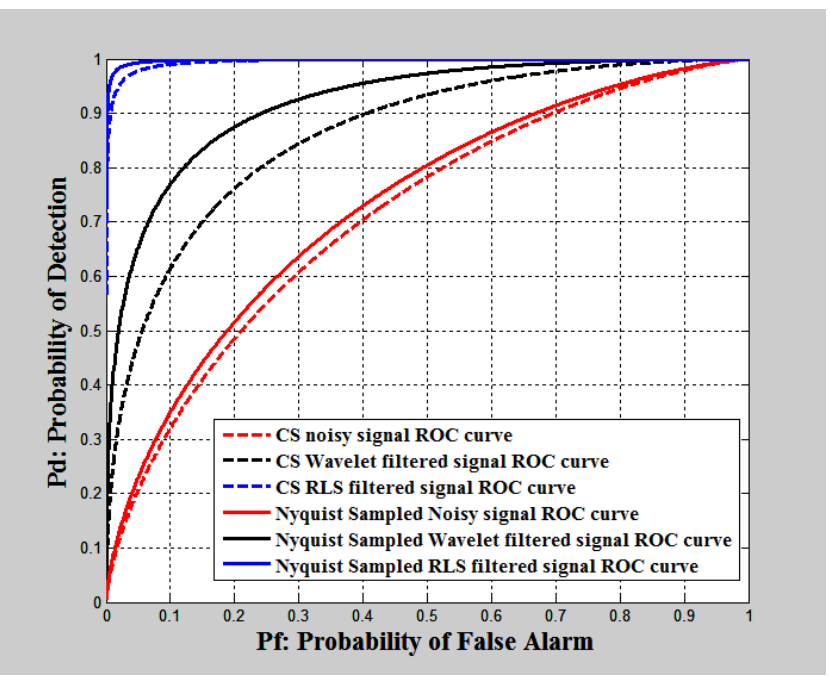

Fig. 9: Comparison between ROC Curves at 40\% Compression Ratio and the Traditional Energy Detector Using Ordinary Sampling.

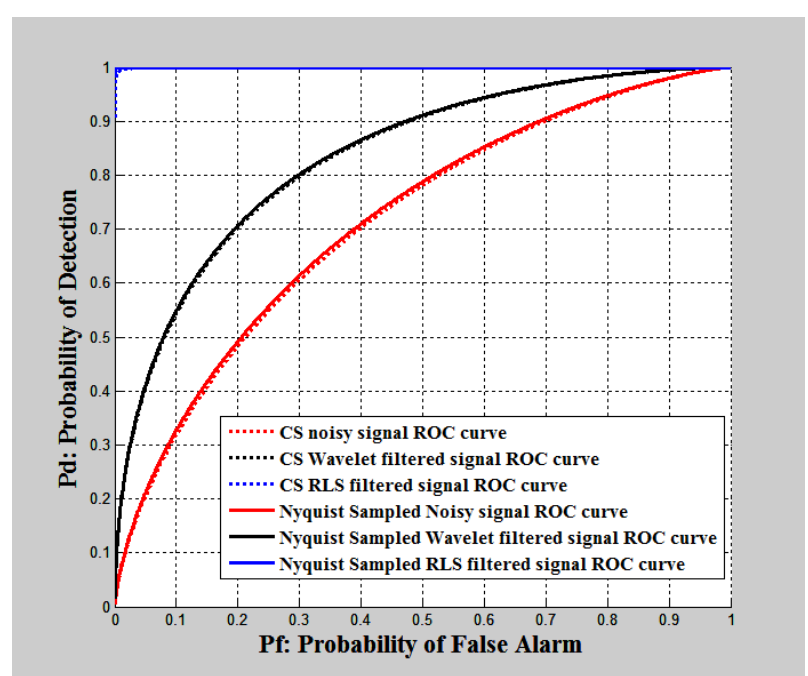

Fig. 10: Comparison between ROC Curves at $60 \%$ Compression Ratio and the Traditional Energy Detector Using Ordinary Sampling. 


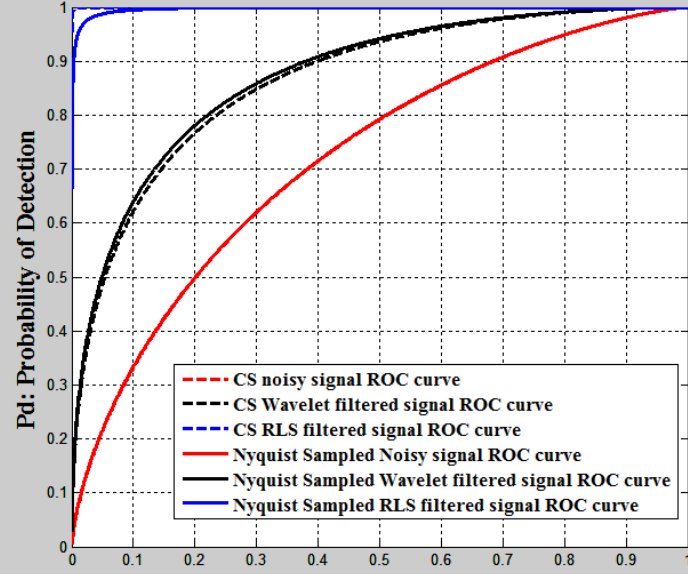

Pf: Probability of False Alarm

Fig. 11: Comparison between ROC Curves at $80 \%$ Compression Ratio and the Traditional Energy Detector Using Ordinary Sampling.

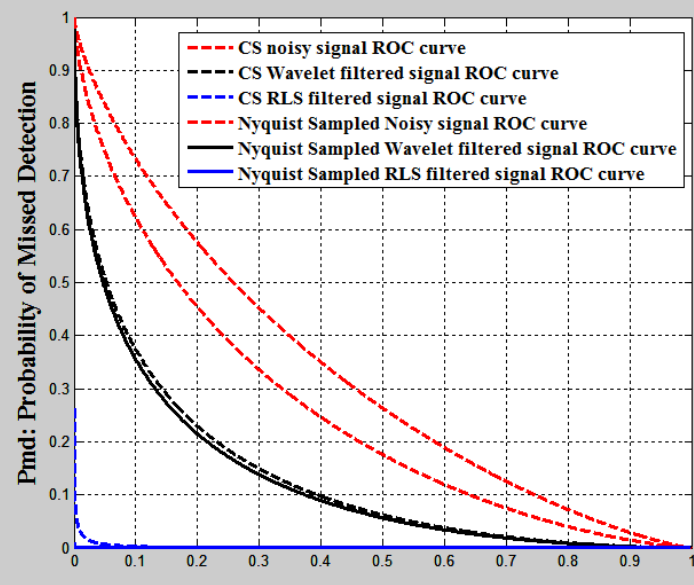

Pf: Probability of False Alarm

Fig. 12: Comparison between ROC Curves at 20\% Compression Ratio and the Traditional Energy Detector Using Ordinary Sampling.

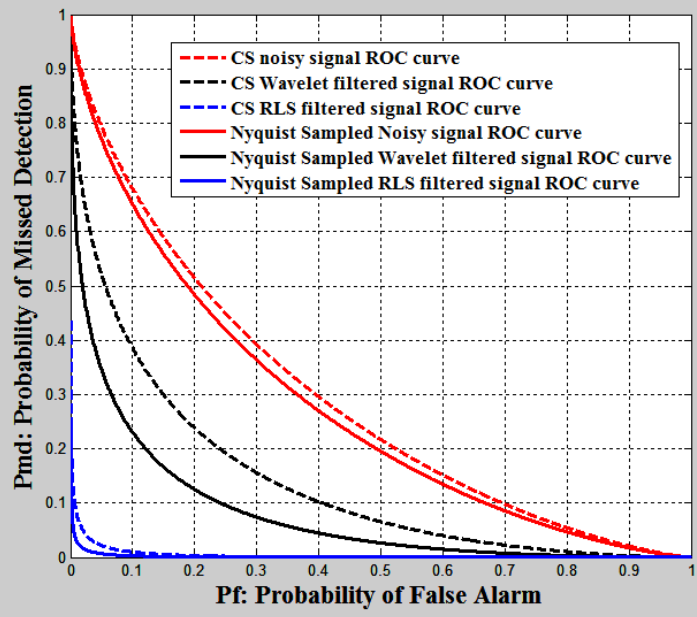

Fig. 13: Comparison between ROC Curves at 40\% Compression Ratio and the Traditional Energy Detector Using Ordinary Sampling.

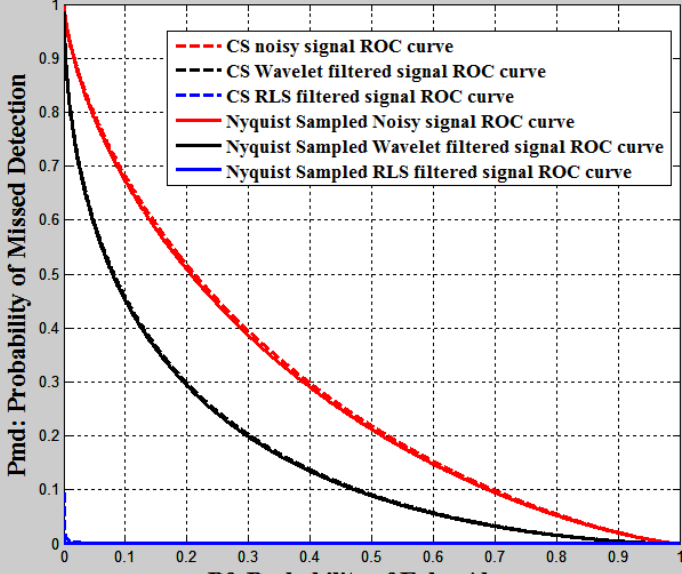

Pf: Probability of False Alarm

Fig. 14: Comparison between ROC at $60 \%$ Compression Ratio and the Traditional Energy Detector Using Ordinary Sampling.

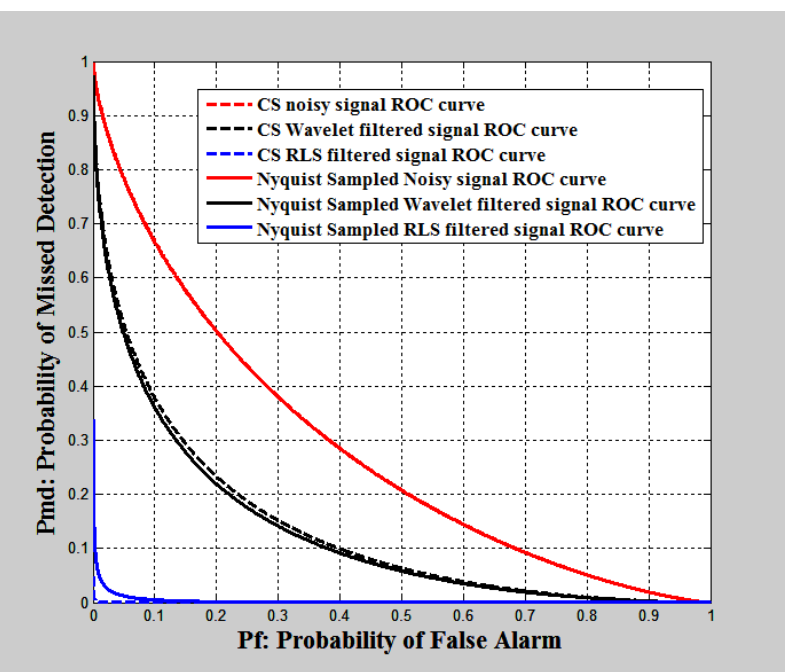

Fig. 15: Comparison between ROC Curves at $80 \%$ Compression Ratio and the Traditional Energy Detector Using Ordinary Sampling.

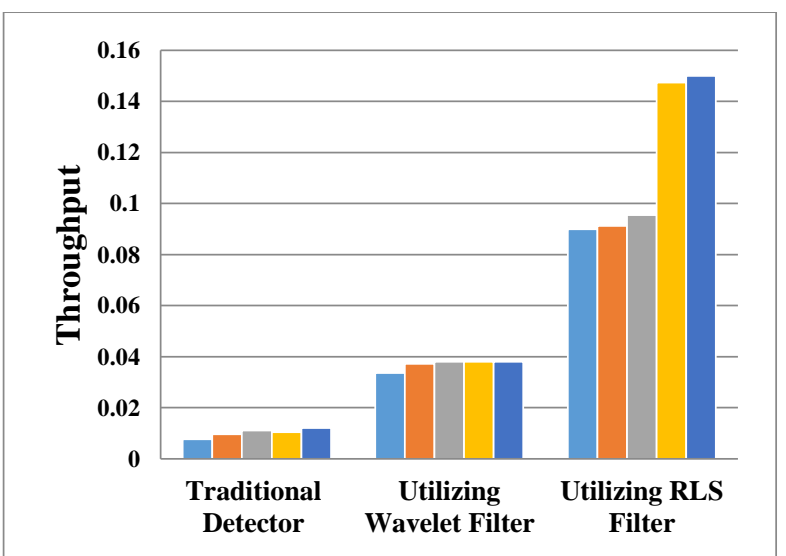

Fig. 16: Throughput Calculations for Traditional Energy Detector and the Proposed Energy Detectors Using Compressive Sensing at Different Compression Ratios. 
Table 2: Throughput Calculations for Traditional Energy Detector and the Proposed Energy Detectors Using Compressive Sensing at Different Compression Ratios $(\mathrm{C} / \mathrm{R})$

\begin{tabular}{|c|c|c|c|c|c|}
\hline & $\begin{array}{l}20 \% \\
\mathrm{C} / \mathrm{R}\end{array}$ & $\begin{array}{l}40 \% \\
\mathrm{C} / \mathrm{R}\end{array}$ & $\begin{array}{l}60 \% \\
\mathrm{C} / \mathrm{R}\end{array}$ & $\begin{array}{l}80 \% \\
\mathrm{C} / \mathrm{R}\end{array}$ & $\begin{array}{l}\text { Nyquist } \\
\text { Sampling }\end{array}$ \\
\hline Traditional Ener- & 0.0077 & 0.0097 & 0.0111 & 0.0104 & 0.012 \\
\hline $\begin{array}{l}\text { Proposed detector } \\
\text { based on CS and } \\
\text { wavelet de- } \\
\text { noising filter }\end{array}$ & 0.0336 & 0.0372 & 0.038 & 0.038 & 0.038 \\
\hline $\begin{array}{l}\text { Proposed detector } \\
\text { based on CS and } \\
\text { RLS de-noising } \\
\text { filter }\end{array}$ & 0.09 & 0.0912 & 0.0955 & 0.1474 & 0.15 \\
\hline
\end{tabular}

Case 3: Proposed energy detector using optimized compression ratio

In this section, the proposed energy detection technique the Genetic Algorithm (GA) optimization tool in MATLAB [26]. The GA estimates the optimal compression ratio that introduces a minimum least mean square error between the absolute values of ROC curves using ordinary sampling and the ROC curves using CS The cost function $(\mathrm{CF})$ to be minimized is written as follows:

$\mathrm{CF}=\sqrt{\frac{1}{\mathrm{~N}} \sum_{\mathrm{i}=1}^{\mathrm{N}}\left|\mathrm{X}_{\mathrm{oi}}-\widehat{\mathrm{X}}_{\mathrm{ci}}\right|^{2}}$

Where $\mathrm{X}_{\mathrm{o}}$ denotes the values of ROC curves using ordinary sampling, $\widehat{X}_{\mathrm{ci}}$ denotes the values of ROC curves using CS and N is the length of the $\mathrm{X}_{\mathrm{oi}}$ or $\widehat{\mathrm{X}}_{\mathrm{ci}}$ vector.

The GA optimization tool options are adjusted as listed in Table 3. The GA tool is run over the pre-assigned compression ratio range $10 \% \leq \mathrm{C} / \mathrm{R} \leq 90 \%$. The setting options of the GA optimization tool in MATLAB are adjusted as listed in table 3. The optimum compression ratio is obtained using only $91 \mathrm{GA}$ iterations and is found to be $\mathrm{C} / \mathrm{R}=64 \%$. The simulation results revealed that the ROC curves using CS at $\mathrm{C} / \mathrm{R}=64 \%$ are highly coincide with the ROC curves using ordinary sampling as shown in Fig.17 and Fig.18.

Table3: The Setting Options of the GA Optimization Tool in MATLAB Used in the Simulation Process.

\begin{tabular}{ll}
\hline Options = & \\
\hline Population Type: & 'doubleVector' \\
PopInitRange: & {$[2 \mathrm{x} 1$ double] } \\
PopulationSize: & 20 \\
CrossoverFraction: & 0.8000 \\
ParetoFraction: & {[]} \\
MigrationDirection: & 'forward' \\
MigrationInterval: & 20 \\
MigrationFraction: & 0.2000 \\
Generations: & 100 \\
TimeLimit: & Inf \\
FitnessLimit: & - Inf \\
StallGenLimit: & 50 \\
StallTimerLimit: & Inf \\
TolFun: & $1.000 \mathrm{e}-006$ \\
TolCon: & $1.000 \mathrm{e}-006$ \\
InitialPopulation: & {[]} \\
InitialScores: & {[]} \\
InitalPenalty: & 10 \\
PenaltyFactor: & 100 \\
PlotInterval: & 1 \\
CreationFcn: & $@$ gacreationunionform \\
FintessScalingFcn: & $@$ fitscalinggrank \\
SelectionFcn: & $@$ selectionstochunif \\
CrossoverFcn: & {$[[1 \mathrm{x} 1$ function_handle] $][1]$} \\
MutationFcn: & {[]} \\
DistanceMeasureFcn: & {[]} \\
HybridFcn: &
\end{tabular}

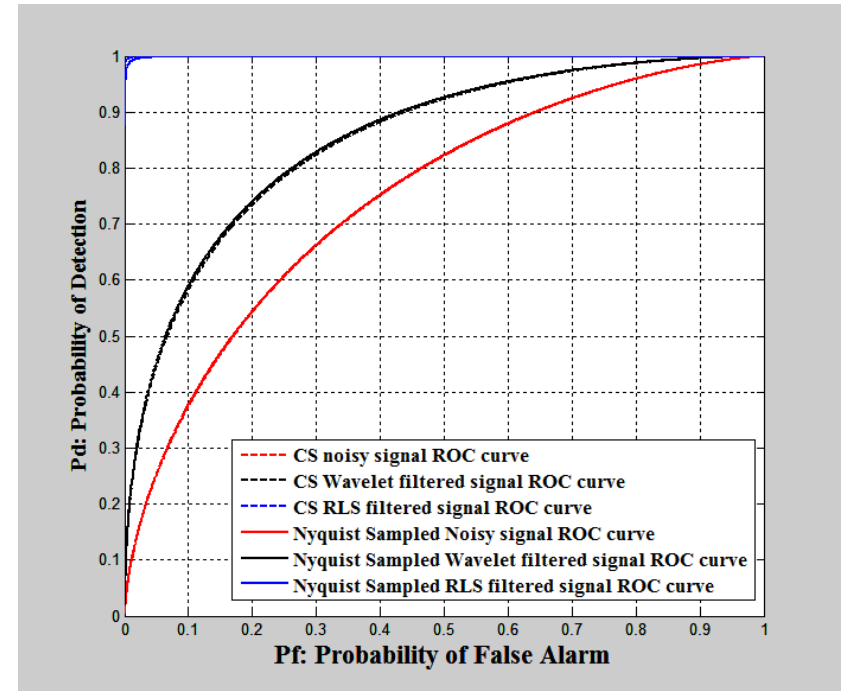

Fig. 17: Comparison between ROC Curves at Optimized Compression Ratio (64\%) and the Traditional Energy Detector Using Ordinary Sampling.

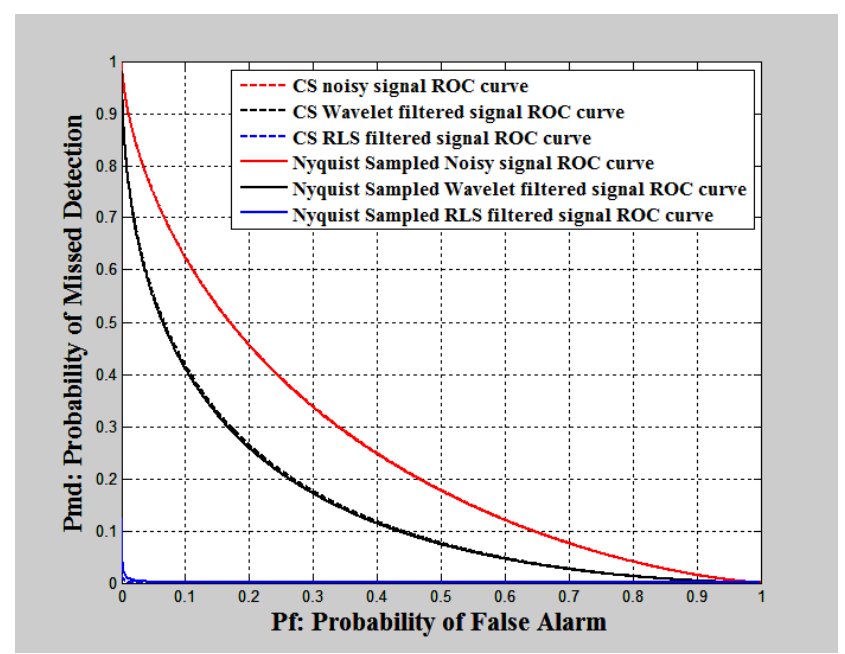

Fig. 18: Comparison between ROC Curves at Optimized Compression Ratio (64\%) and the Traditional Energy Detector Using Ordinary Sampling.

\section{Conclusions}

In this paper, efficient spectrum sensing technique based on energy detection, CS, and de-noising techniques are introduced. The simulation results revealed RLS de-noising filter provides much higher performance more than the energy detector based on wavelet de-noising filter and the traditional energy detector. In addition, the performance-enhancement effect of the energy detector has greatly reflected on the throughput of the system. The throughput calculations show that after applying the Wavelet and RLS denoising filters are increased to 3.166 and 12.5 times greater than the throughput of the traditional detector respectively. Furthermore, simulation results indicate that the throughput is lower than the optimal throughput achieved using ordinary sampling. However, it is worth noting that for compression ratios greater than $60 \%$, the curves using CS become very close to the ROC curves using the ordinary sampling. Finally, a proposed energy detection technique using optimized compression ratio is introduced using $\mathrm{Ge}-$ netic Algorithm (GA) optimization tool. The simulation results revealed that the ROC curves using $\mathrm{CS}$ at compression ratio equal to $64 \%$ are highly coinciding with the ROC curves using ordinary sampling. 


\section{References}

[1] P. SembaYawada, An Jian Wei and M. Mbyamm Kiki, "Performance evaluation of energy detection based on non-cooperative spectrum sensing in cognitive radio network," 2015 4th International Conference on Computer Science and Network Technology (ICCSNT), Harbin, China, 2015.

[2] Performance of Spectrum Sensing in Cognitive Radio",I.J. Information Technology and Computer Science, 2012, 11, 11-17.

[3] Hussein Al-Mood, H. S. Al-Raweshidy,"Energy Detection Performance Enhancement for Cognitive Radio Using Noise Processing, Approach", IEEE, 2013.

[4] Al-Hmood H. and Al-Raweshidy H. S., "Energy detection performance enhancement for cognitive radio using noise processing approach," Global Information Infrastructure Symposium - GIIS 2013, Trento, pp. 1-6, 2013.

[5] Emmanuel Candes and Justin Romberg, "Practical signal recovery from random projections", SPIE Symposium on Electronic Imaging, 2005.

[6] Donoho, "Compressed sensing", IEEE Transactions Info Theory, 52(4):12891306, 2006

[7] Z. Tian and G. B. Giannakis, "Compressed sensing for wideband cognitive radio", in ICASSP, vol. 4, pp.1357-1360, April 2007.

[8] Z. Tian and G. B. GiannakisPeh, E.C.Y.;Anh Tuan Hoang, "Sensing-Throughput Tradeoff for Cognitive Radio Networks ", Wireless Communications, IEEE Transactions on, vol.7, no.4, pp.1326,1337, April 2008.

[9] S. S. Chen, D. L. Donoho, and M. A. Saunders, "Atomic decomposition by basis pursuit", SIAM journal on scientific computing, vol 20, no. $1, \quad$ pp. 1998 https://doi.org/10.1137/S1064827596304010.

[10] E. J. Candes and T. Tao, "Decoding by linear programming, IEEE Transactions on Information Theory", SIAM journal on scientific computing, vol. 51, no. 12, pp. 42034215, 2005.

[11] F. Ghido, I. Tabus, "Sparse Modeling for Lossless Audio Compression,",IEEE Trans. on Audio, Speech, and Language Processing, vol.21, no.1, pp.14,28, 2013.

[12] H.M. Kasem and M. Elsabrouty,"Perceptual compressed sensing and perceptual sparse fast fourier transform for audio signal compression,", in; fifteenth International Workshop on Signal Processing Advances in Wireless Communications (SPAWC),2014, pp.444-448. https://doi.org/10.1109/spawc.2014.6941862.

[13] J. Skilling and S. Gull, "Algorithms and applications, IEEE Transactions on Information Theory", in Maximum-entropy and Bayesian methods in inverse problems, pp. 83132, Springer, 1985. https://doi.org/10.1007/978-94-017-2221-6 5.

[14] Y. C. Pati, R. Rezaiifar, and P. Krishnaprasad, "Orthogonal matching pursuit: Recursive function approximation with applications to wavelet decomposition, IEEE Transactions on Information Theory", in Signals, Systems and Computers, pp. 4044, IEEE, 1993.

[15] G. Davis, S. Mallat, and M. Avellaneda, "Adaptive greedy approximations, Constructive approximation, ", in IEEE Transactions on Information Theory Signals, Systems and Computers, vol. 13, no. 1 , pp. 5798, 1997.

[16] Miller, "Subset selection in regression", iCRC Press, 2002.

[17] R.Gribonval and M. Nielsen, "Highly sparse representations from dictionaries are unique and independent of the sparseness measure", iComputational Harmonic Analysis, vol. 22, no. 3, pp. 335355 , 2007.

[18] "CVX: Matlab software for disciplined convex programming, version 2.0". I. CVX Research, http://cvxr.com/cvx, Aug. 2012.

[19] M. Grant and S. Boyd, "Graph implementations for nonsmooth convex programs", in Recent Advances in Learning and Contro (V. Blondel, S. Boyd, and H. Kimura, eds,Lecture Notes in Control and Information Sciences, pp. 95110, SpringerVerlag Limited, 2008.

[20] Emmanuel C.Ifeachor,BarrieW.Jervis, "Digital Signal Processing, A Practical Approach ",. Second edition 2002, chapter (10).

[21] M. B. Sousa e Silva and A. N. Barreto, "Spectrum sensing in cognitive radio networks through change detection technique ".. I Telecommunications Symposium (ITS), 2014 International, Sao Paulo, 2014, pp. $1-5$.

[22] GutoQuanXiang,YuXia Zhang, "Analysis of RLS Adaptive Filter in Signal De-noising ",. TElectrical and Control Engineering (ICECE), 2011 International Conference on, Yichang.2011, pp. 5754-5758.

[23] David L. Donoho and Iain M. Johnstone, "Minimax estimation via wavelet shrinkage", Technical report, 1992.
[24] T. E. Bogale, L. Vandendorpe and L. B. Le, "Sensing throughput tradeoff for cognitive radio networks with noise variance uncertainty,"2014 9th International Conference on Cognitive Radio Oriented Wireless Networks and Communications (CROWNCOM), Oulu, 2014.

[25] "IEEE Standard of Information Technology" Part 22: Cognitive Wireless RAN Medium Access Control (MAC) and Physical Layer (PHY) Specifications: Policies and Procedures for Operation in the TV Bands, 1 July 2011

[26] R. L. Haupt and D. H. Werner Piscataway, "Genetic Algorithms in Electromagnetics", NJ: IEEE PressWiley-Interscience, 2007. https://doi.org/10.1002/047010628X. 